Halté Pierre

Docteur en Sciences du Langage

Université de Lorraine, Université du Luxembourg

CREM

Pierre.halte@orange.fr

\title{
ENJEUX PRAGMATIQUES ET SÉMIOTIQUES DE L'ÉTUDE DES ÉMOTICÔNES
}




\section{INTRODUCTION}

Les émoticônes sont apparues relativement récemment dans la communication médiatisée par ordinateur, notamment dans les conversations par t'chat. Très peu étudiées, dans l'ensemble, jusqu'alors, elles représentent pourtant un enjeu considérable dans la remise en question nécessaire de certains paradigmes des sciences du langage (les rapports entre le verbal / non verbal, la question du sens propositionnel des énoncés, le statut du signe graphique, etc.). Il s'agit de signes graphiques imitant des mimiques faciales ou d'autres objets, indiquant une émotion du locuteur, qui interagissent avec des énoncés - le plus souvent propositionnels - pour produire un sens qui dépasse celui, littéral, de la proposition logique énoncée. Elles rentrent donc dans le cadre de la modalisation et plus généralement des marques de subjectivité dans le discours. Notre objectif est ici d'interroger cet objet d'étude sous deux angles : d'abord, celui de la sémiotique, afin de mieux comprendre à quoi nous avons affaire. Cette première approche nous fournira les bases nécessaires pour aborder les conséquences qui découlent nécessairement du statut sémiotique des émoticônes : leurs fonctions pragmatiques et énonciatives.

Nous proposerons d'abord une définition ainsi qu'un rapide historique de ces signes. Nous les analyserons ensuite sous l'angle de la sémiotique peircienne et de celle des rapports entre texte et images. Enfin, nous observerons des exemples tirés d'un corpus de conversations par t'chat ${ }^{1}$, établi lors de notre travail de thèse (Halté 2013), pour dégager quelques fonctions pragmatiques et énonciatives des émoticônes et interroger, à partir des conclusions tirées, quelques notions fondamentales comme celle de modalisation ou de portée.

\section{DÉFINITION ET HISTOIRE DE L’ÉMOTICÔNE}

\section{Définition préliminaire}

Les émoticônes sont des icônes de mimiques faciales dont la fonction principale est d'indiquer une émotion. On les appelle aussi parfois «smileys» ou encore «binettes». Les émoticônes sont à l'origine constituées des signes issus du code ASCII («American Standard Code for Information Interchange »), qui est la plus ancienne norme de codage des caractères courants en informatique. On y retrouve donc 128 caractères usuels de l'écrit: lettres de l'alphabet romain, chiffres, signes de ponctuation, etc ${ }^{2}$. De cet ensemble de signes naît une pratique spécifique de l'informatique: l'(autoproclamé) art ASCII, qui consiste à utiliser ces caractères pour représenter divers objets, personnages, animaux, etc. Exemple : voici un oiseau réalisé en art ASCII :

$\backslash \_/ 0<$

Le grand public place sous le terme d'émoticônes l'ensemble des icônes s'intégrant aux énoncés verbaux lors d'une communication médiée par ordinateur, quelle qu'elle soit (chat, sms, etc.); nous appliquerons ici le terme d'émoticône uniquement à des icônes dont la fonction est d'être l'indice d'une émotion ou d'une attitude subjective portant sur l'énonciation d'un contenu' ${ }^{3}$. Ainsi, dans tout ce

\footnotetext{
${ }^{1}$ Notre corpus daté de 2009 a été constitué en nous connectant au hasard dans un salon de discussion, en utilisant le logiciel mIRC, et en laissant les conversations se dérouler sans intervention de notre part pendant cinq heures environ. Nous avons ensuite récolté les historiques des conversations, sauvegardés automatiquement par le logiciel. Pour plus d'informations, voir Halté 2013.

${ }^{2}$ Pour plus d'informations sur l'ASCII, consulter la page wikipedia y étant consacrée : http://fr.wikipedia.org/wiki/American_Standard_Code_for_Information_Interchange\#Table_des_128_caract.C3. A8res_ASCII

${ }^{3}$ Voir la seconde partie de l'article, « Sémiotique de l'émoticône », pour plus d'informations sur ce qu'est un indice.
} 
qui suivra, nous ne considèrerons pas les assemblages de signes de l'alphabet ASCII comme l'icône d'oiseau ci-dessus, comme des émoticônes : sa fonction n'est pas de montrer l'émotion du locuteur. Par contre, le sourire (ou «smiley ») : «:)», qui se lit en penchant la tête vers la gauche comme toutes les émoticônes occidentales ${ }^{4}$ imitant des mimiques faciales, relève de l'émoticône dès lors qu'il sert à ajouter une couche de subjectivité au sens d'un énoncé verbal.

\section{Petite histoire de l'émoticône}

Au-delà des recherches historiques, sérieuses ou moins sérieuses, se posant la question de la présence d'une émoticône dans un discours d'Abraham Lincoln en 1862 (Lee, 2009), les plus anciennes occurrences d'émoticônes sont généralement considérées comme contemporaines des premières interactions par réseau informatisé ayant eu lieu aux alentours des années 1970, aux États-Unis, grâce au réseau «PLATO », qui constitue certainement la première communauté en ligne connue (pour plus de renseignements, voir Dear, 2002). Cependant, on trouve des exemples de formes typographiques destinées à coder les émotions telles qu'elles apparaissent sur un visage humain dès 1881, dans un article consacré à l'art typographique du journal Puck.

La complexité de la communication via Internet rend très difficile la vérification des informations concernant l'apparition des émoticônes, et la plupart du temps la «doxa» des internautes attribue l'origine des émoticônes telles que nous les connaissons aujourd'hui à ce mail de 1982, écrit par un universitaire (Scott Fahlman) utilisant le réseau de son université pour communiquer avec ses pairs :

19-Sep-82 11:44 Scott E Fahlman :-)

From: Scott E Fahlman <Fahlman at Cmu-20c>

I propose that the following character sequence for joke markers:

:-)

Read it sideways. Actually, it is probably more economical to mark things that are NOT jokes, given current trends. For this, use

$:-($

Je propose la séquence de caractères suivante comme marqueur de plaisanterie :

:-)

À lire de côté. En fait, c'est probablement plus économique de marquer les trucs qui ne sont PAS des plaisanteries, compte tenu des tendances actuelles. Pour ce faire, utilisez

$:-\left({ }^{5}\right.$

Cette hypothèse paraît intuitivement plus faible que celle qui attribue l'origine des émoticônes au réseau PLATO, parce qu'il semble très peu probable qu'une occurrence isolée, énoncée comme une règle par un individu, devienne effectivement si largement employée. Il est à notre avis plus réaliste de considérer qu'un faisceau d'emplois au sein d'une communauté de locuteurs a contribué à installer

\footnotetext{
${ }^{4}$ Les émoticônes dites « orientales » peuvent se lire de face : « $\left({ }^{\wedge} \wedge\right)$ », par exemple.

${ }^{5}$ Nous traduisons.
} 
l'émoticône à la place - très importante et en pleine expansion - qu'elle occupe aujourd'hui. Notons enfin que si, à l'origine, les émoticônes sont constituées des caractères ASCII, aujourd'hui, dans les réseaux sociaux et même dans les traitements de texte, taper la suite de caractères «:) », par exemple, transforme automatiquement cette suite en pictogramme, comme suit : «() ». Plus spectaculaire, il existe aussi dans certains chats et dans certains forums une fonction permettant de remplacer une séquence de caractères donnée par une émoticône, automatiquement : une séquence donnée, placée entre deux doubles points, par exemple «: lol :», est automatiquement remplacée par un pictogramme de visage rieur. C'est un exemple de la correspondance reconnue par les modérateurs des chats et des forums entre des marques modales diverses : les interjections acronymiques (comme « lol», acronyme de «lots of laugh » ou de «laughing out loud», employé pour montrer le rire du locuteur) et les émoticônes. La séquence peut aussi contenir un adjectif, par exemple «: triste: » sera remplacé automatiquement par un pictogramme représentant un visage triste.

Dans l'univers de la téléphonie mobile et de la communication par SMS, il n'existe aujourd'hui plus que des pictogrammes. Les émoticônes faites de signes de ponctuation n’y ont quasiment plus cours, même si, curieusement, certaines marques de téléphones portables proposent encore des banques d'émoticônes constituées de signes de ponctuation. Ceci dit, ces dernières sont automatiquement transformées en pictogrammes dans les messages dans lesquels elles apparaissent. Certains sites vendent d'ailleurs des «banques d'émoticônes» plus ou moins originales, classant parfois les émoticônes par thème («Amour », «Alcool », etc).

Ces émoticônes à vendre sont parfois animées, et peuvent être récupérées par les utilisateurs, afin de les employer sur des sites de réseaux sociaux, par exemple. L'animation apporte évidemment une grande variété aux émoticônes, ainsi que la possibilité d'intégrer des pictogrammes et non plus des signes de ponctuation. Ces banques d'émoticônes ont un grand succès auprès des plus jeunes, qui les utilisent à l'excès. Ceci étant dit, les émoticônes les plus employées sont toujours les plus simples, comme l'émoticône de sourire ou de tristesse, ou encore le clin d'œil ; et le fait de toujours pouvoir utiliser les signes de l'alphabet ASCII, directement accessibles sur le clavier et automatiquement remplacés par des pictogrammes animés ou pas, permet que les émoticônes faites de signes de ponctuation restent très utilisées car elles sont très pratiques et rapides à produire via un clavier. Toutes les émoticônes dérivent d'un système sémiotique fondé sur l'utilisation d'un clavier. Ce sont bien les contraintes qu'impose l'utilisation d'un clavier, ainsi que la conversation par t'chat qui ont conduit à élaborer des signes permettant de charger affectivement des énoncés verbaux que l'écrit rendait un peu trop impersonnel. Il va de soi qu'un utilisateur écrivant avec un stylo n'aurait certainement jamais produit une émoticône constituée de signes de ponctuation, comme «;) », par exemple, mais aurait certainement dessiné un petit visage souriant d'un trait de crayon. Même si les émoticônes sont aujourd'hui presque systématiquement remplacées par des pictogrammes, animés ou pas, elles portent encore dans leur forme les conséquences des contraintes exercées par le genre de discours dont elles sont issues, le chat, avec son interface, ses usages, ainsi que par l'outil qui permet de s'exprimer dans ce contexte : le clavier et les signes qui sont inscrits sur ses touches.

L'histoire du terme " émoticône » est encore plus difficile à reconstituer que l'histoire du signe en luimême. Le nom «émoticône », en tout cas, apparaît bien après les signes eux-mêmes (son apparition est très vaguement datée : entre 1985 et 1994). Il est impossible de trouver un article ou une recherche ayant proposé initialement ce terme pour désigner ce type de signe. Le terme émoticône, combinant de manière intuitivement assez juste « émotion » et «icône », sert cependant à désigner des signes qui ne sont pas des icônes d'émotions en tant que telles. L'émoticône imitant un clin d'œil, par exemple, «;) », n'imite pas une émotion au sens strict. Behague (2008), dans son travail de thèse intitulé Analyse multimodale de l'émotion dans un discours convaincant, s'attache tout d'abord à définir ce qu'est traditionnellement une émotion :

Émotion vient du latin emotum forme nominale du verbe latin emovere, « mouvoir hors de », «émouvoir » ( Dictionnaire Larousse de la langue Française, p.623). Ce cadre étymologique souligne bien à quel point l'agitation physique et psychique qui s'empare du sujet et qui le projette en quelque sorte hors de lui est de l'ordre de la "déconvenue ». Dans un discours le movere, la mobilisation des âmes et des coeurs, l'emporte sur toute autre considération : le discours doit être émouvant, ému, passionnant et 
passionné. L'émotion se situe entre le physique et le psychique pour contrôler les désirs, les pulsions primitives et pour mieux faire valoir le sens de l'éthique (ethos) avec celui de la raison (logos). L'émotion se caractérise globalement comme utile à une situation donnée qui requiert des réponses comportementales expressives et intentionnelles. Elle est empiriquement investigable, généralement transitoire et d'une certaine intensité. Elle se manifeste au niveau linguistique de différentes manières : choix des mots, intonation, exclamation (Caffy, Janney, 1994). (Behague, 2008, p. 30).

Dans cette définition, les indices qui permettent de percevoir ou de montrer une émotion (comme les manifestations linguistiques évoquées à la toute fin de la citation) ne se confondent pas avec l'émotion elle-même. Ainsi, le sourire peut indiquer qu'on éprouve de la joie, mais il n'est pas l'émotion ellemême, il n'en est qu'un des indices. On peut faire l'hypothèse qu'un faisceau d'indices fonctionnant ensemble est ce qui permet d'interpréter une émotion.

Les sciences cognitives (la psychologie surtout) proposent par la suite une définition que Behague reprend :

Elles [les émotions] se caractérisent par un ensemble de « réponses physiologiques comportementales expressives et cognitives expérentielles concomitantes »(Luminet, 2002, p.46). Suivant cette approche, les émotions reflètent un ensemble de réponses adaptatives qui servent de signal à l'organisme. Il n'existe qu'un ensemble restreint d'émotions de base (colère, peur, tristesse, dégoût, surprise, joie). Elles sont manifestement universelles et perdurent grâce à la présence de déclencheurs spécifiques (par exemple : perte d'un être cher pour la tristesse). Contrairement à l'humeur, l'émotion se caractérise par sa durée courte, quelques secondes ou minutes et, des éléments déclencheurs, aisément identifiables. Nous pouvons retenir que l'émotion "est une séquence de changements d'états intervenant dans les cinq systèmes organiques dont les composantes sont le système cognitif (là où passe l'évaluation cognitive), le système neurophysiologique, le système moteur (les muscles), le système relationnel (car il y a des tendances qui sont créées par l'émotion) et, le système moniteur donc la surveillance (pour moi, c'est le sentiment). » (Scherer, 2001). (Behague, 2008, p. 31)

Il est difficile de faire coïncider une émotion avec ce qui manifeste sa présence. Il peut paraître étrange de séparer les émotions de ce qui indique leur présence, même si c'est le postulat qui est adopté dans ces définitions. En effet, on voit mal comment définir une émotion en dehors des signes qui la constituent. On pourrait faire l'hypothèse que l'émotion n'a pas d'existence immanente, indépendante des signes qui l'accompagnent, mais qu'au contraire ces signes constituent l'émotion. C'est un large débat qui ne sera pas abordé ici. Pour Yus (2011), citant Dresner \& Herring (2010, p. 252), il est abusif de lier les émoticônes aux émotions :

L'émoticône est une des façons les plus connues de connoter du texte écrit avec une information visuelle non-verbale (aussi appelée « smiley ») (voir Quinion 1996, Witmer \& Katzman 1997). Les plus courantes sont «joie » ou «bonheur »: [:-)], «tristesse »: [:-(] et «clin d'œil [;-)]. Elles étaient initialement inhérentes à la communication via les salons de chat mais ont été exportées aux messageries instantanées, aux SMS, à Twitter et aux sites de réseaux sociaux, entre autres. L'origine du terme, une combinaison entre «émotion» et «icône », peut induire en erreur. Comme Dresner \& Herring (2010: 252) le clarifient à juste titre, l'objectif des émoticônes n'est pas seulement d'exprimer les émotions de l'utilisateur, puisque «beaucoup d'émoticônes ne semblent pas exprimer une seule émotion, voire semblent n'en exprimer aucune. Est-ce qu'un visage dont la langue est sortie, par exemple ;-p est le signe d'une émotion spécifique ? Des sources variées lui attribuent un sens tel que la taquinerie, le flirt, ou le sarcasme, et tous peuvent être associés à des états émotionnels, mais ne sont pas des émotions à proprement parler. Ou encore, considérons le clin d'œil ;-) : conventionnellement, il indique que le scripteur plaisante, mais les plaisanteries ne sont pas associées à un seul état émotif. On peut plaisanter quand on est heureux ou triste. » (Yus, 2011, p.166, nous traduisons) 
Dans tous les cas, on peut retenir que si l'on considère ces définitions " classiques » de l'émotion, on ne peut pas dire que les émoticônes imitent les émotions, tout au plus peut-on dire que les émoticônes, interagissant avec le co(n)texte, peuvent être des indices, parmi d'autres, d'un état émotionnel général, sans en être forcément des marqueurs spécifiques. Les émoticônes, contrairement à ce qu'indique leur nom, n'imitent pas des émotions, mais des mimiques faciales ou des gestes qui sont habituellement des indices de telle ou telle émotion. Les datations fournies par les différentes sources s'intéressant aux émoticônes proposent des périodes de temps très larges (1985-1994), sans qu'il soit possible d'en savoir plus. Notons que pendant longtemps, c'est le terme de «smiley » qui a été utilisé pour désigner toutes les émoticônes, ce que l'on peut légitimement considérer comme un emploi abusif du terme, « smiley » ne désignant littéralement que le sourire.

\section{SÉMIOTIQUE DE L’ÉMOTICÔNE}

\section{L’émoticône : symbole, indice, icône ? Peirce à la rescousse}

Le modèle sémiotique de Peirce, tel qu'il est décrit par Everaert-Desmedt (1990), permet d'expliquer la manière dont les émoticônes font sens. Nous prenons ici le parti de nous en tenir à l'interprétation très fonctionnelle d'Everaert-Desmedt du modèle sémiotique peircien, qui permet de catégoriser les signes linguistiques de façon à la fois claire et dynamique : icône, indice et symbole sont des concepts qui y sont bien définis.

L'intérêt principal du modèle sémiotique peircien est qu'il fonctionne en trios plutôt que de façon binaire comme celui de Saussure (les deux chercheurs élaborent d'ailleurs leurs modèles presque simultanément). La production de sens, pour Peirce, c'est « un processus triadique, qui met en relation un signe ou representamen $\left(1^{\mathrm{er}}\right)$, un objet $\left(2^{\mathrm{e}}\right)$, et un interprétant $\left(3^{\mathrm{e}}\right) »$ (Everaert-Desmedt, 1990, p.26). On a la figure suivante :

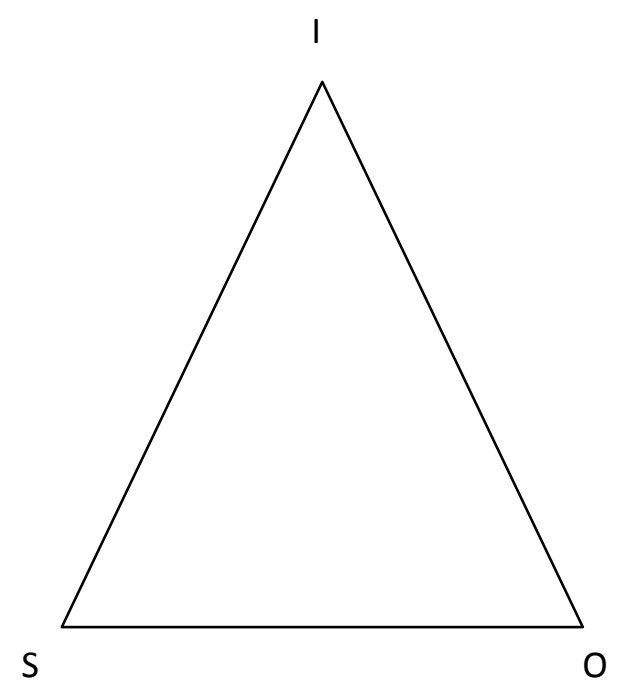

Figure 1 : Modèle sémiotique de Peirce

On le voit, selon Peirce, il n'y a pas de production de signification en dehors de la pragmatique, «c'est-à-dire d'une action dans un contexte » (Everaert-Desmedt, 1990, p. 29) : un signe, selon lui, est «le sujet d'une relation triadique avec un second appelé son objet, pour un troisième appelé son interprétant »(Peirce 1931-1935 t1, p.117, cité par Everaert-Desmedt, 1990, p.27).

Les relations entre ces trois composantes (Signe, Objet, Interprétant) fonctionnent, chacune, de trois manières différentes. Chacune des trois composantes de ce triangle se décompose en trois types : il existe ainsi trois types d'objet, trois types d'interprétant, et trois types de signes. De même, chaque relation entre les pointes du triangle peut être de trois types. Nous ne nous intéresserons ici qu'aux 
trois types de signes qui existent, et aux trois différents types de rapports entre le signe - dans notre cas, l'émoticône - et l'objet du monde.

Pour Peirce, il existe trois types de signe : le «qualisigne», «signe dont le fondement est une qualité »; le «sinsigne », qui est « une chose ou un évènement réel, spatio-temporellement déterminé, qui fonctionne comme signe » (Everaert-Desmedt, 1990, p. 51); et enfin le «légisigne », « signe dont le fondement est une loi »(Everaert-Desmedt, 1990, p. 51), une convention. Il est impossible d'illustrer un qualisigne autrement que par l'imitation ; c'est une perception immédiate de la qualité d'un objet, avant qu'on la transforme par un processus de pensée. Le sinsigne est un signe tel qu'il se manifeste dans un espace-temps donné. Par exemple, une grimace est un sinsigne indiquant que, ici et maintenant, son émetteur souffre. Le légisigne est un signe conventionnel, qui fait partie d'un système. Tous les signes de la langue sont des légisignes. Notons au passage qu'évidemment, tout légisigne ne peut se manifester qu'au travers de sinsignes qu'Evereaert-Desmedt et Peirce appellent des « répliques » de légisigne :

Tout légisigne signifie par son application dans un cas particulier, qu'on peut appeler sa réplique: par exemple, le mot «the » qui apparaît d'ordinaire en anglais quinze à vingt-cinq fois par page. Il est à chaque fois le même mot, le même légisigne. Chaque cas particulier est une réplique. La réplique est un sinsigne. Ainsi, tout légisigne requiert des sinsignes. Mais ces derniers ne sont pas des sinsignes ordinaires, comme le sont des reproductions particulières qui sont considérées comme signifiantes. Et la réplique ne serait pas signifiante sans la loi qui la rend signifiante. (Peirce, 1978)

Ainsi, quand je parle en ce moment, ma voix et les mots que j'utilise sont bien ancrés dans le réel, dans un «ici et maintenant», et indiquent - au minimum- que je parle. J'utilise des répliques de légisignes : des mots codés selon des conventions, faisant partie d'un vaste système conventionnel, qui se manifestent dans des sinsignes, ici et maintenant. Avec les émoticône, nous avons affaire à des légisignes qui se manifestent en sinsignes : l'émoticône est bien un signe conventionnel qui fait partie d'un système codé, dont certaines composantes peuvent être interchangées pour donner des sens différents (par exemple, le passage d'une parenthèse fermée à une parenthèse ouverte change une émoticône de sourire en émoticône de tristesse)

Pour Peirce, la relation entre le signe et l'objet se fait de trois manières :

Iconique: «Un signe renvoie à son objet de façon iconique lorsqu'il ressemble à son objet». (Everaert-Desmedt, 1990, p.53). Le signe imite dans sa forme l'objet du monde. Le pictogramme d'oiseau, vu dans la première partie, puisqu'il imite une mimique faciale, est iconique.

Indicielle ou indexicale : «L'indice est un fait immédiatement perceptible qui attire l'attention sur son objet, lequel n'est pas immédiatement perceptible. » (Everaert-Desmedt, 1990, p.61); on parle d'indice lorsqu'une partie du discours manifeste dans la langue la situation d'énonciation de son locuteur. Les déictiques, par exemple, sont des indices. L'émoticône est un indice : c'est bien un fait immédiatement perceptible (l'occurrence de l'émoticône) qui attire l'attention sur un objet qui n'est pas perceptible immédiatement, ici l'émotion du locuteur.

Symbolique : «Un signe est un symbole lorsqu'il renvoie à son objet en vertu d'une règle, d'une loi, d'une association d'idées générales. » (Everaert-Desmedt, 1990, p.65). La plupart des mots et des structures prédicatives phrastiques sont symboliques : ils renvoient, de manière arbitraire, à leur référent. Ainsi, le mot «chien », dans l'énoncé «Le chien est beau», par exemple, renvoie de façon arbitraire à l'objet du monde « chien ».

Pour récapituler notre approche du modèle de Peirce nous proposons ce schéma : 


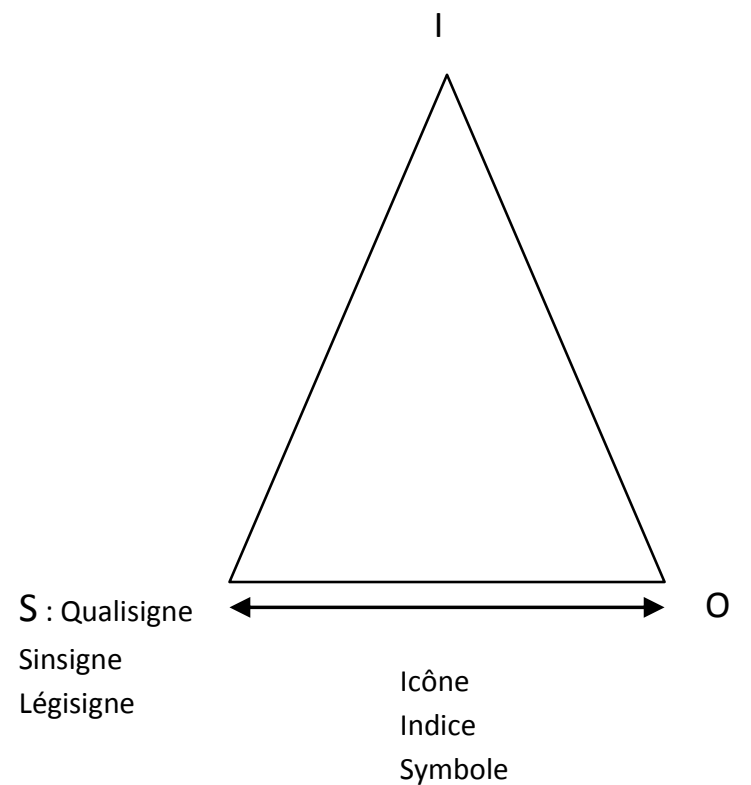

Figure 2 : Modèle sémiotique de Peirce

Un qualisigne ne peut être qu'iconique : la seule manière de représenter la saisie immédiate d'une réalité, c'est l'imitation de cette réalité. Un sinsigne peut être iconique (une maquette) ou indiciel (un cri). Un légisigne peut être iconique («cocorico»), indiciel (tous les déictiques, comme «je») ou symbolique (les noms communs comme « pomme», par exemple).

C'est l'usage que l'interprétant fait du signe, la façon dont il le relie à l'objet du monde, qui détermine s'il s'agit d'une icône, d'un indice ou d'un symbole. Ainsi, une onomatopée comme « cocorico » peut être considérée soit comme une icône, lorsqu'elle est utilisée pour imiter le cri du coq, soit comme un indice, lorsqu'elle est utilisée par le locuteur pour manifester sa fierté d'être français. Il en va de même pour nos pictogrammes : dès lors qu'ils sont des simples représentations graphiques pour le locuteur, comme le pictogramme d'oiseau de la première partie ils sont des icônes ; par contre, lorsqu'ils deviennent indices d'une émotion, ce sont des indices, et c'est sur ce critère que nous fondons la catégorie des émoticônes. Parfois, le même signe peut être icône et indice, tour à tour, ou en même temps : dans le cas de l'émoticône de sourire, c'est parce qu'elle imite une mimique faciale (elle en est donc l'icône) qu'elle peut indiquer une émotion (elle en devient l'indice). N'oublions pas que Peirce est un des socles philosophiques sur lesquels s'appuiera la pragmatique lorsqu'elle naîtra dans les années 1950, et que c'est donc, chez Peirce comme en pragmatique, la pratique du sujet qui prime dans la détermination de la sémiose.

Pour récapituler, les émoticônes sont donc pour nous, suivant le parcours sémiotique de Peirce, des légisignes, qui sont des icônes de mimiques faciales ou d'autres éléments, et qui, de par ce statut d'icône, peuvent facilement devenir des indices d'émotions. Le nom est donc bien trompeur: l'émoticône n'est pas l'icône d'une émotion, elle en est l'indice. Il existe des cas plus complexes que les simples émoticônes faciales, qui mettent en jeu les trois aspects de la relation entre signe et objet envisagés par Peirce ; par exemple, l'émoticône « $\langle 3$ ». C'est l'icône d'un cœur, le cœur étant luimême symbole (c'est-à-dire un signe arbitrairement, selon une loi humaine, lié à son objet) de l'amour. L'émoticône « <3» devient donc l'indice de l'amour éprouvé par le locuteur en suivant un parcours sémiotique complexe : c'est parce qu'elle est l'icône, l'imitation d'un symbole, qu'elle peut devenir l'indice de ce à quoi est attaché le symbole. 


\section{L'émoticône entre texte et image}

Une des caractéristiques principales des émoticônes de notre corpus est qu'elles sont constituées de signes issus de l'alphabet ASCII, signes qui sont par ailleurs employés comme signes de ponctuation, ou comme lettres. Ainsi, le signe de parenthèse fermée de «:)», ne peut pas être lu dans une émoticône comme le signe indiquant la fin d'une parenthèse, mais doit bien être interprété de façon iconique, comme une image, une représentation iconique d'une bouche dont les coins sont relevés. De même, le « $\mathrm{D}$ » de « :D », émoticône représentant de façon iconique un visage rieur, ne peut pas être lu comme une lettre, associant la graphie « $\mathrm{D} »$ au son [d], mais doit être interprété comme une image représentant la bouche ouverte et rieuse du locuteur. Voici comment Klinkenberg décrit, pour ce qui concerne les caractéristiques iconiques de l'écriture, cette double sémiotique des signes de la langue :

L'interpénétration entre le signe scriptural et le signe iconique se laisse donc décrire de la manière suivante : un même stimulus (empirique) renvoie simultanément à deux signifiants, l'un de nature iconique et l'autre de nature scripturale. En termes de rhétorique visuelle, ces interpénétrations produisent des figures du type «chafetière » (cf Groupe M, 1992), avec cette différence que les deux ensembles en intersection ne sont pas tous deux constitués de stimuli correspondant à des déterminants iconiques : c'est le cas de l'un, l'autre étant constitué d'objets graphiques. (Klinkenberg 2008, p. 10)

Ainsi, dans nos exemples, le signe «parenthèse fermée » est-il un stimulus qui peut renvoyer à deux signifiants (nous verrons dans la partie suivante comment le modèle de Peirce permet de penser la distinction de Klinkenberg entre stimulus et signifiant) : soit la parenthèse appartient au paradigme scriptural des signes de ponctuation, indiquant la fermeture d'une parenthèse, soit elle appartient au paradigme des signes iconiques, imitant dès lors une bouche aux coins relevés. De même, la lettre « D » constitue-t-elle un stimulus pouvant renvoyer à plusieurs signifiants : soit elle appartient au paradigme de la langue, et elle sert à coder le son [d], entre autres fonctions linguistiques, soit elle appartient à un paradigme de signes iconiques, et elle sert alors à représenter de façon iconique une large bouche rieuse, par exemple. Notons que les deux paradigmes évoqués (signes de la langue et signes iconiques) ici reposent tous deux sur des conventions, même si les règles qui les régissent sont différentes.

Les émoticônes, cependant, sont des signes assez simples. Il s'agit toujours de les interpréter de façon iconique, et elles constituent un système à part entière, fermé. Elles ne laissent donc pas la place à des interprétations doubles : jamais, lors de la lecture d'une émoticône, il ne sera pertinent de lire le signe «parenthèse fermée " comme l'indice de la fermeture d'une parenthèse, contrairement à ce qui se passe avec certains signes de l'ancien égyptien, décrits ci-dessus par Klinkenberg, qui peuvent à la fois être interprétés comme faisant partie d'un paradigme linguistique ET d'un paradigme iconique :

Il s'agit d'un phénomène que l'on trouve dans toutes les cultures et dont la tradition remonte à la plus haute antiquité. On peut ainsi la voir fonctionner en ancien égyptien, dans ces cas où l'icône et l'idéogramme - deux entités distinctes en droit — se superposent presque exactement. C'est ce que montre telle gravure pariétale, qui met en scène le rite de l'union des deux pays : un même végétal peut y être lu tantôt comme icône des fleurs caractérisant la Haute- et la Basse-Égypte, tantôt (ou simultanément) comme hiéroglyphe à valeur phonétique ( $m h w$ et $S w a w$, « Haute-Égypte » et « BasseÉgypte »). De nos jours, cette fonction se voit aussi fréquemment utilisée en publicité. (Klinkenberg 2009, p. 11)

La parenthèse fermée, ou le «D», contrairement à ces signes de l'ancien égyptien évoqués par Klinkenberg, ne sont donc jamais simultanément interprétés comme une icône ET comme un signe de ponctuation ou une lettre : c'est soit l'un, soit l'autre. 
Cela n'empêche pas la prononciation orale de certaines émoticônes, comme « XD », représentant iconiquement un visage rieur, prononcé parfois à l'oral en se servant des phonèmes représentés par les lettres : [iksde] : il s'agit tout simplement dans ce cas d'être conscient de la double sémiotique des signes qu'on utilise, et de jouer là-dessus. Ce n'est pas parce qu'il n'est pas pertinent d'interpréter une lettre comme représentant un son lors de la lecture d'une émoticône que l'utilisateur ignore le fait que ce même signe puisse représenter un son, ce qui lui permet tout à fait de jouer sur les deux tableaux à la fois, en oralisant une succession de signes qui sont normalement à interpréter de façon iconique, comme «XD ». Pour interpréter [iksde], prononciation orale de l'émoticône « XD », correctement, il faut absolument connaître le paradigme sémiotique des émoticônes, ses règles, ses usages, les éléments qui le constituent : sinon, il est impossible de savoir que la séquence de lettres nommées à l'oral peut être lue, dans un certain contexte de production, comme une icône, et il est impossible de comprendre qu'il s'agit de montrer que l'on rit; un locuteur qui ignorerait tout des émoticônes ne pourrait pas interpréter correctement [iksde], il y reconnaîtrait bien évidemment les noms des lettres « $\mathrm{X}$ » et « $\mathrm{D}$ », mais il chercherait certainement, par exemple, à savoir de quoi ce « $\mathrm{X}$ » et ce « $\mathrm{D}$ » sont les initiales, alors qu'il faut comprendre que la prononciation de « XD » renvoie à la graphie des lettres, et chercher ce dont cette graphie est l'icône. La prononciation des noms des lettres ou des signes de ponctuation (on pourrait en effet tout à fait imaginer un locuteur énonçant par jeu, à l'oral, « deux points parenthèse fermée » pour désigner les signes qui constituent une émoticône de sourire et s'attribuer l'émotion liée à cette émoticône) composant une émoticône n'est qu'un jeu sur les paradigmes sémiotiques, paradigmes qu'il faut connaître pour pouvoir jouer. Il s'agit simplement de désigner un signe par d'autres signes, ce qui n'enlève pas au premier signe son statut de signe iconique.

\section{ÉMOTICÔNE ET ÉNONCIATION}

\section{Inscription du sujet dans son discours ou l'émoticône comme marque d' « ancrage énonciatif »}

Par «ancrage énonciatif » nous entendons ici la façon dont le locuteur se met en scène dans son propre discours, avec une intensité qui est perceptible grâce à des indices variés, dont les émoticônes font partie. Il s'agira ici surtout d'analyser les possibilités de l'intégration des émoticônes au discours rapporté.

Il est évident que l'émoticône joue un rôle fort dans l'ancrage énonciatif du locuteur, puisqu'elle est toujours l'indice de l'émotion ou de la subjectivité du locuteur, elle inscrit nécessairement ce dernier de façon forte, en montrant sa présence explicite de sujet éprouvant, dans son discours. Ce statut d'indice pose cependant certains problèmes, notamment en ce qui concerne le discours rapporté. Impossible de les utiliser au discours direct :

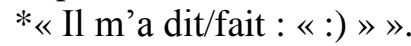

On n'en trouve aucun exemple dans le corpus. Encore moins au discours indirect :

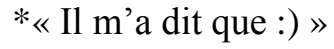

ou même

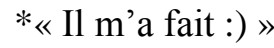

ne sont pas possibles. Dans les faits, les émoticônes accompagnent souvent des énoncés qui sont du discours rapporté, notamment indirect libre, mais indiquent toujours l'émotion du locuteur «sujet parlant », du locuteur du discours citant, et pas celui du discours cité :

(1) [15:12] <MeeYung> je suis en train de discuter avec une coréenne adoptée ... et il semblerait que la plupart des coréens adoptés naissent avec une hépatite ...

[15:12] < MeeYung> et que durant des années ça peut être dormant mais quand ça se réveille c'est très très compliqué :s

Ici, <MeeYung> rapporte le discours d'une coréenne adoptée avec qui il discute. Ainsi, l'énoncé « et que durant des années ça peut être dormant mais quand ça se réveille c'est très très compliqué » rapporte le discours de cette personne coréenne ; par contre, l'émoticône qui suit cet énoncé, « :s », est 
nécessairement comprise comme l'indice de l'attitude subjective de $<$ MeeYung $>$ par rapport à cet énoncé, et pas comme celui de l'attitude subjective de la coréenne dont le discours est rapporté ici. L'émoticône ramène invariablement le locuteur à la matérialité de la situation d'énonciation ; une émoticône peut difficilement être enchâssée.

Dans l'exemple suivant se pose le problème de l'intégration d'une émoticône à un récit comportant du discours rapporté : 06[15:21]* @ Candy pratique une échographie sur pedri`

[15:21] <Bourguideche21>c'est un garcon ou une fille?

$[15: 22]<@$ Candy $>$ ya deux jumeaux la dedans

$[\ldots]$

[15:22] <Bourguideche21> minimum

[15:22] <pedri > la derniere fois qu'on m'en a fait une, le mec m'a demandé si j'étais alcoolique -_-'

[15:22] <LesPrie> mdr pedri', ha ouais, dur

[15:22]<@Candy>bah je peut écrire debout LesPrie

[15:22] <pedri门 > ouais.... il m'a demandé si je buvais beaucoup... -_-'

Dans cet exemple, l'émoticône " -_-'» accompagne un énoncé verbal qui raconte l'échographie de l'utilisateur <pedri>. Ce dernier écrit qu'à cette occasion, le médecin s'occupant de l'examen lui a " demandé s'il était alcoolique ». La question est de savoir à qui attribuer l'émoticône qui accompagne cet énoncé. Il va de soi que ce n'est pas au médecin, pour les raisons évoquées précédemment : même dans le cas d'un discours rapporté au style indirect, comme ici, l'émoticône est toujours attribuée au locuteur du discours citant. C'est donc bien à <pedri> qu'est attribuée l'émoticône. Mais la question est de savoir, alors, s'il s'agit d'indiquer avec cette émoticône l'émotion de <pedri> au moment du récit, ou alors au moment de l'histoire : cette émoticône indique-t-elle que le locuteur <pedri>, au moment où il énonce ce récit, est blasé, ou alors est-elle une représentation d'un <pedri> « virtuel », passé, éprouvant cette émotion au moment où on lui fait son échographie ? Les deux ! Dans les deux cas, il s'agit de la même émotion, éprouvée par le même locuteur ; c'est à notre avis parce que l'émoticône est à la fois un indice de l'émotion passée du locuteur, et de l'émotion qu'il ressent au moment de l'énonciation, qu'elle permet d'ancrer le locuteur à la fois dans le contenu de son récit et dans le fait de le raconter, ce qui permet de l'impliquer très fortement dans son espace énonciatif. Le locuteur se met en scène en train de rééprouver une émotion qu'il a éprouvée au moment du récit ; sa mise en scène coïncide donc avec ce qu'il montre au moment de l'énonciation, il montre qu'il revit ici et maintenant l'émotion éprouvée au moment du récit. Ce faisant, l'émoticône assure la continuité entre la temporalité du récit, et celle du moment énonciatif qui le contient.

L'analyse de ces exemples montre que l'émoticône permet au locuteur de se mettre en scène, en tant que sujet, dans son propre discours, de façon efficace. L'émoticône ne peut pas être employée au sein du discours rapporté sans être nécessairement attribuée au sujet parlant, contrairement à l'interjection qui peut tout à fait être le fait du locuteur du discours cité. Cette caractéristique permet au locuteur de mettre en avant le plan de l'énonciation plutôt que celui de l'énoncé, et donc d'affirmer son rôle dans la production de l'énoncé de manière forte et marquée. De plus, cette caractéristique permet aussi de jouer, lors notamment de récits à la première personne, avec la temporalité du récit et celle de l'histoire, puisque l'émoticône est susceptible à la fois de montrer l'émotion du locuteur au moment de son récit et au moment de son énonciation, cette coïncidence indiquant et renforçant la crédibilité du récit.

\section{Modalisation}

L'émoticône établit toujours des relations avec les énoncés qui suivent, ou qui précèdent, modifiant, par inférences, l'interprétation qui en est faite. On ne trouve d'ailleurs pas, dans notre corpus, d'exemple d'émoticône produite de façon complètement indépendante. Certes, elles peuvent constituer des interventions à elles seules, mais ces interventions sont toujours, sauf peut-être dans le cas où l'émoticône de sourire accompagne une formule de salutation, des réactions à des énoncés verbaux, impliquant un effet de reprise du contenu qui précède, et il est impossible de commencer une conversation, ex nihilo, par une émoticône, sans que l'interlocuteur et les autres utilisateurs du salon 
de discussion ne cherchent «sur quoi elle porte» ou ce qui a provoqué son énonciation, et fassent porter sur ce qu'ils trouvent à l'issue de cette recherche la subjectivité indiquée par l'émoticône. Les émoticônes relèvent donc de la modalisation (voir Perrin 2012, Vion 2004, Bally 1944, etc.) : elles relèvent du modus, c'est-à-dire de l'attitude subjective inhérente à la production de tout énoncé, et portent sur un dictum, ce que décrit l'énoncé de façon vériconditionnelle (autrement dénommé « contenu propositionnel »). Elles montrent, et ne disent pas, en d'autres termes.

Nous nous poserons donc tout d'abord la question de l'émoticône en tant que modalisateur, de façon tout à fait «traditionnelle », c'est-à-dire en considérant les émoticônes dont la fonction spécifique est de modifier l'interprétation d'un contenu propositionnel en faisant porter sur lui la subjectivité du locuteur. Rappelons ici la définition proposée par Vion, dans laquelle il propose de distinguer entre modalité et modalisation de la façon suivante :

La modalisation n'est donc pas assimilable à la modalité qui, nous l'avons vu, contribue directement au sémantisme des énoncés auxquels elle participe. Nous constatons, au contraire, que sa présence opacifie le sens dans la mesure où la production d'un commentaire réflexif, portant sur le dire ou le dit, contribue à construire l'image d'un sujet dédoublé et donc d'un énoncé qui ne peut être interprété de manière directe. Émettre un commentaire réflexif sur un énoncé donne à penser que cet énoncé ne va pas de soi et ne saurait être interprété comme un énoncé plus ordinaire. La modalisation entraîne une complexification de la représentation construite par le locuteur ainsi qu'une opacification du sémantisme et un « brouillage » au niveau de ses positionnements. (Vion, 2004, p. 103)

Pour Vion, on parle donc de modalité quand un élément de l'énonciation modifie directement le sémantisme de l'énoncé, c'est-à-dire quand cet élément porte sur le contenu propositionnel (le dictum si l'on suit la typologie de Bally, ou encore le «dit») et le modifie : dans «Pierre viendra certainement jeudi prochain » (Vion, 2004, p. 102), l'adverbe « certainement » agit directement sur la proposition énoncée dans « Pierre viendra jeudi prochain ».

On parle de modalisation lorsque l'élément modal n'agit pas directement sur le contenu propositionnel de l'énoncé qu'il accompagne, mais est plutôt un commentaire réflexif sur l'énonciation elle-même, ce qui provoque ce que Vion appelle un «dédoublement énonciatif». Ainsi, dans «Je suis certain que Pierre viendra jeudi » (Vion, 2004, p. 102), dira-t-on que « je suis certain » relève de la modalisation : cette partie de l'énoncé ne modifie pas le contenu propositionnel de «Pierre viendra jeudi » mais fournit un cadre surplomblant, subjectivant, à cette proposition. C'est à notre avis tout à fait ce qui se produit lors de la plupart des emplois d'une émoticône monologique dans le chat; Vion, d'ailleurs, sans évoquer l'émoticône, propose l'exemple suivant pour illustrer ce qu'est la modalisation : "Pierre viendra jeudi prochain (avec des signes coverbaux de certitude) ». L'émoticône semble tout à fait être un de ces signes coverbaux servant à montrer une modalisation et donc correspondre à la définition de la modalisation proposée par Vion.

Observons quelques exemples sous cet angle. Dans l'exemple (3), l'émoticône se comporte bien comme un modalisateur «à la Vion»: elle ne modifie pas le contenu propositionnel de l'énoncé verbal qu'elle accompagne, mais fournit plutôt un cadre surplombant et subjectivant qui « opacifie » (Vion 2004, p. 102) le sens de cet énoncé, fournissant une information du type «cet énoncé dit quelque chose qu'il ne faut pas comprendre littéralement mais plutôt comme l'indique l'émoticône ».

(3) $[12: 52]<$ wassila|Caoua > Woucky

[12:52] <wassila|Caoua> tes vraiment naze

[12:53] <Woucky> wassila|Caoua, j'te fous dans mon ignore list, définitivement. :)

L'émoticône de sourire ne rentre pas " dans le sémantisme » de l'énoncé verbal qu'elle accompagne, mais elle fournit bien un cadre subjectif qui surplombe l'énoncé. Il y a bien ici un dédoublement énonciatif tel que l'envisage Vion: d'une part, un énoncé qui dit et décrit quelque chose, d'autre part, une émoticône qui opacifie en quelque sorte le sens de cet énoncé, qui indique qu'il ne faut pas seulement le comprendre littéralement. C'est toute la fonction de l'émoticône dans cet exemple que de modifier la façon dont est interprété le contenu propositionnel : en effet, il n'est pas possible de comprendre cette émoticône comme un symptôme naturel, résultant 
nécessairement de l'état de fait qui est décrit par l'énoncé. Menacer quelqu'un, ou décrire une action préjudiciable à quelqu'un, ne déclenche pas le sourire ni, a fortiori, la production d'une émoticône de sourire. Cette émoticône a donc pour rôle de révéler la position subjective du locuteur par rapport au contenu qu'il énonce, afin de modifier l'interprétation globale de l'énoncé. Dans ce cas, l'émoticône fait partie de l'énoncé; elle en révèle le modus. Notons au passage que dans la lecture et l'interprétation des ensembles «énoncé verbal + émoticônes ", l'étude de Provine et al (2007) montre que le lecteur lit toujours l'énoncé verbal en premier, puis l'émoticône ; il produit ensuite seulement une interprétation « rétroactive » tenant compte de l'effet de l'émoticône sur le contenu propositionnel. Le cas de (4) est différent :

(4) $[15: 00]<\%$ Spinelli $>$ ca va ca vient...

[15:00] $<\%$ Spinelli $>$ mais bon en ce moment ca vient pas trop :S

[15:00]<\%ondes-virtuelles $>$ :(

La question qui se pose, pour (4), est de savoir si l'émoticône de mimique faciale triste produite par <ondes-virtuelles> peut être considérée comme un modalisateur ou pas. Il s'agit d'une émoticône produite en réaction à un énoncé produit par un autre locuteur, et la fonction de l'émoticône n'est pas de modifier l'interprétation littérale du contenu propositionnel énoncé par l'autre, mais bien, plutôt, de présenter une réaction subjective à l'état de fait que décrit ce contenu. Est-il possible de modaliser un énoncé produit par un autre que soi ?

Si l'on suit la définition de Vion, , la réponse est non : si la modalisation est la fonction qui consiste à conduire l'interlocuteur à interpréter un énoncé de façon non littérale, alors elle ne peut être cantonnée qu'à un seul locuteur, ou plutôt un seul sujet parlant pour reprendre le terme de Ducrot. On peut montrer son attitude subjective par rapport à un contenu propositionnel élaboré par un autre que soi (c'est d'ailleurs en partie ce qui se passe dans (4)); par contre, pour faire en sorte que cette monstration modifie l'interprétation littérale d'un énoncé, il faut nécessairement que le contenu propositionnel énoncé, et l'attitude subjective montrée portant sur cet énoncé, proviennent du même sujet parlant. C'est pourquoi, dans le cas de (4), et toujours dans la conception de Vion, l'émoticône ne peut pas être considérée comme un modalisateur, même si elle révèle l'attitude subjective du locuteur par rapport à un énoncé. Il y a ici, à notre avis, une remarque à faire sur la façon dont Vion envisage la modalisation : il nous semble tout à fait possible de faire porter une attitude subjective sur un énoncé produit par autrui, et peu importe que cela en modifie l'interprétation littérale ou non. C'est pourquoi nous considérons, comme Perrin, que la modalisation porte toujours sur l'énonciation d'un contenu, sans poser la question de l'interprétation littérale ou non de l'énoncé. Dans cette perspective, une émoticône dialogique peut tout à fait être considérée comme un modalisateur : on considèrera simplement que produire une émoticône à la suite d'un énoncé produit par l'interlocuteur implique une reprise implicite de la proposition énoncée, sur laquelle le locuteur fait porter une subjectivité qu'il montre grâce à l'émoticône.

Plus problématique encore, les cas où l'émoticône porte non pas sur un contenu, sur ce qui est énoncé, mais sur une énonciation, une «façon de dire », comme, par exemple, dans (10) :

(5) $[13: 12]<$ Marcovanbouten> re

[13:12] < Marcovanbouten> slt Bourguideche

[13:12] < Marcovanbouten> bissss Candy

[13:12] <Marcovanbouten> slt_Roi2Coeur

[13:12] <Marcovanbouten> :)

Dans cet exemple, on peut faire l'hypothèse que l'émoticône de sourire produite par $<$ Marcovanbouten> porte sur le fait qu'il répète les salutations, plutôt que sur les salutations ellesmêmes. Il s'agit toujours d'une modalisation, seulement, plutôt que de viser l'énonciation du contenu, elle vise le fait de répéter l'énonciation d'un contenu. Cela fait toujours partie du processus énonciatif. On a donc bien, toujours, une émoticône qui porte sur l'énonciation d'un contenu. 
Par cette émoticône de sourire, <Marcovanbouten $>$ veut simplement indiquer qu'il enchaîne les salutations pour s'amuser. Il présente son amusement à la fois comme le résultat de son propre jeu énonciatif, mais aussi comme sa source. Ce type d'exemple ouvre un questionnement profond sur la façon de concevoir la modalisation. Notre position est que la modalisation porte toujours sur l'énonciation d'un contenu, ce qui recouvre différents aspects des processus de production d'énoncés : on modalise non seulement l'énonciation d'un contenu, mais aussi le fait de répéter une énonciation, la façon d'énoncer, toutes composantes de l'énonciation... Il n'y a donc pas nécessairement de changement de niveau, ni, dans le cas où plusieurs modalisateurs se trouvent côte à côte (comme nous l'avons vu dans certains exemples type « ah lol :) ») de modalisation portant sur une modalisation : il y a d'une part l'énonciation d'un contenu, d'autre part, une attitude subjective que le locuteur montre, à l'aide de divers procédés qui peuvent se combiner entre eux, concernant l'énonciation de ce contenu. La force énonciative des émoticônes tient à cette caractéristique : elles peuvent viser n'importe quel élément de l'énonciation d'un contenu, et parfois même plusieurs à la fois. Il est en effet parfois difficile d'établir sur quelle composante porte l'émoticône, comme dans l'exemple (6) :

(6) $[15: 30]<$ Angel> Bisouxxxxxxxxxxx ma kinder bueno Candy

[15:30] <Angel> Bisoux LesPrie

[15:30]<@Oceans> kiss Angel

[15:30] <Angel> Kissous Oceans

[15:31] <Bourguideche> rooo c'es meugnon tout cet amour ${ }^{\wedge} \wedge$

[15:31] < Angel> Lol Bourguideche $\mathrm{c}$ bon de se savoir aimée :p

$[15: 31]<$ Bourguideche> visiblement ${ }^{\wedge} \wedge$

Comment décider si l'émoticône «^^», produite par <Bourguideche> après l'énoncé " rooo c'est meugnon tout cet amour », porte sur l'énonciation du contenu propositionnel de cet énoncé ( $<$ Bourguideche $>$ voulant montrer que ce qu'il énonce doit être compris non littéralement mais comme une plaisanterie), ou alors sur l'énonciation des multiples salutations qui précèdent $(<$ Bourguideche $>$ signalant son amusement devant le fait que tout le monde se salue en utilisant des formules plus ou moins amusantes), ou enfin n'est qu'une représentation iconique du locuteur voulant montrer qu'il éprouve de la joie, symptomatique, devant certains éléments de la situation d'énonciation? L'ambiguïté tient à une question de portée. L'émoticône porte à la fois la potentialité de ces trois valeurs, et c'est justement pour ça qu'elle est particulièrement pertinente dans ce type d'énoncé. On peut modaliser l'énonciation d'une séquence plus vaste qu'un acte simple.

L'émoticône est donc sans aucun doute, pour nous, une marque modale : elle révèle toujours l'attitude subjective, émotionnelle, du locuteur. Elle est aussi, la plupart du temps, un modalisateur au sens de Perrin: elle porte toujours sur un élément ou sur la globalité de l'énonciation d'un contenu propositionnel, ce qui peut d'ailleurs conduire à distinguer deux notions : celle de portée (le segment syntaxique sur lequel porte l'émoticône), et celle de visée (l'élément de la situation d'énonciation visé par la modalisation : acte de langage, forme de l'énoncé, contenu propositionnel... $)^{6}$.

\section{CONCLUSION : DU NON-VERBAL À L'ÉCRIT?}

Les deux points précédents montrent à notre avis à quel point l'émoticône est un enjeu de taille pour les études pragmatiques et les sciences du langage en général. Elle permet en effet des effets énonciatifs complexes et relève de la modalisation, comme d'autres marques modales, par exemple les interjections et certains adverbes. La différence entre les émoticônes et ces dernières est, cependant, qu'elles n'appartiennent pas du tout au même paradigme : les émoticônes ne relèvent pas de la langue verbale, ce ne sont pas des mots comme les interjections, mais des imitations graphiques de mimiques faciales. Pourtant, elles sont présentes à l'écrit, au contact de la langue. Elles sont donc le point d'entrée d'une réflexion globale sur les sciences du langage, et de leur remaniement. Elles montrent en effet qu'il faut peut-être cesser de considérer la communication humaine comme, avant tout, logocentrée. Elles permettent de faire disparaître les frontières entre une langue considérée

\footnotetext{
${ }^{6}$ Pour plus d'informations à ce sujet, voir Halté (2013).
} 
traditionnellement comme faisant sens avant tout de manière référentielle, logique, et relevant donc d'études linguistiques fondées sur cette conception, et celle d'un mode de communication multimodal, faisant intervenir, certes, un mode de référence logique et descriptif, mais en constante interaction avec des données qui sont d'un tout autre ordre : données expérientielles, gestes, corps en mouvements ; les deux aspects de la communication n'ayant pas à être nécessairement hiérarchisés. La communication humaine ne fait pas toujours sens d'un point de vue logique, et le sens que nous fabriquons sans cesse ne repose pas seulement sur des processus de référence et de description, de relation entre les mots d'un côté et les choses de l'autre. Notre travail de thèse montrait à quel point les émoticônes sont proches, d'un point de vue sémiotique et pragmatique, d'autres marques modales, les interjections, qui, elles, sont sans aucun doute possible des mots de la langue. Il est donc clair que non seulement les émoticônes montrent que certaines productions «non-verbales » peuvent remplir les mêmes fonctions que les productions «verbales », mais que l'inverse est aussi vrai : certains mots de la langue, apparaissant dans le dictionnaire et considérés comme faisant partie du paradigme verbal de la langue, fonctionnent dans certaines conditions tout à fait comme des gestes ou des mimiques faciales. Il n'est pas étonnant que les signes permettant d'arriver à ces conclusions soient apparus dans un contexte bien particulier, celui des conversations synchrones en ligne (ou chat) dans lequel se mêlent la spontanéité de l'oral aux contraintes de l'écrit. Continuer à étudier ces nouveaux modes de communications, ainsi que les signes qui les peuplent, présente pour toutes ces raisons un intérêt fondamental pour les sciences du langage. 


\section{BIBLIOGRAPHIE}

ANIS J., dir., (1999), Internet, communication et langue française, Paris, coll. « Science Publications », Hermes.

ANIS J., ZARA (2005), «L'ordinateur support textuel ? Le texte informatique comme processus » in L'Ecriture entre support et surface, Paris, L'Harmattan.

BALLY Ch. (1944), Linguistique générale et linguistique française, 2e édition, Berne, A. Francke.

BEHAGUE D. (2008), Analyse multimodale de l'émotion dans un discours convaincant, Paris, Université Paris 8.

DEAR B.L. (2002), PLATO emoticons, accessible uniquement en ligne : http://www.platopeople.com/emoticons.html.

DRESNER E., HERRING S.C. (2010), «Functions of the nonverbal in CMC : Emoticons and illocutionary force », in Communication Theory, $\mathrm{n}^{\circ}$ 20, Munich, Thomas Hanitzch, pp. 249-268.

DUCROT O. (1980), Les mots du discours, Paris, Minuit.

EVERAERT-DESMEDT N. (1990), Le processus interprétatif : introduction à la sémiotique de Ch. S. Peirce, Liège, Mardaga.

HALTE P. (2013), Les marques modales dans les chats : analyse sémiotique et pragmatique des interjections et des émoticônes dans les conversations synchrones en ligne, thèse de doctorat soutenue à Luxembourg, Metz/Luxembourg, Bibliothèque Universitaire de Metz, consultable en ligne : http://www.theses.fr/2013LORR0308

KLINKENBERG J.-M. (2008), «La relation texte-image. Essai de grammaire générale », in Bulletin de la Classe des Lettres, Académie royale de Belgique, 6/19, pp. 21-79. Article disponible en ligne: http://gemca.fltr.ucl.ac.be/docs/cahiers/20090128_Klinkenberg.pdf

LEE J. (19 janvier 2009), «Is That an Emoticon in 1862? », in New York Times, New York, accessible en ligne : http://cityroom.blogs.nytimes.com/2009/01/19/hfo-emoticon/.

\section{MARCOCCIA M.,}

- (1998), «La normalisation des comportements communicatifs sur Internet : Étude sociopragmatique de la netiquette », in Communication, société et internet, Paris, L'Harmattan, pp. 15-32.

- (2000a), «Les smileys, une représentation iconique des émotions dans la communication médiatisée par ordinateur », in Les émotions dans les interactions, Lyon, Presses universitaires de Lyon.

- (2000b), «La représentation du nonverbal dans la communication écrite médiatisée par ordinateur », in Communication et organisation, mis en ligne le 27 mars 2012, accessible en ligne: http://communicationorganisation.revues.org/2431

- (2003), «La communication médiatisée par ordinateur : problèmes de genres et de typologie », in Journée d'études: les genres de l'oral, Lyon, Laboratoire Tech-Cico, Université de Technologie de Troyes.

- (2004), «La communication médiatisée par ordinateur : faire du face à face avec de l'écrit », in Journée d'étude de l'ATALA "Le traitement automatique des nouvelles formes de communication écrite (emails, forums, chats, SMS, etc.) », Troyes, ISTIT/CNRS. 
MARCOCCIA M., GAUDUCHEAU N. (2007), « Le rôle des smileys dans la production et l'interprétation des messages électroniques », in La langue du cyberespace : de la diversité aux normes, Paris, L'Harmattan, pp. 279-295.

PEIRCE Ch. S. (1978), Écrits sur le signe, rassemblés, traduits et commentés par Gérard Deledalle, Paris, Éditions du Seuil.

PERRIN L. (2012), «L'énonciation dans la langue. Ascriptivisme, pragmatique intégrée et sens indiciel des expressions », in Atayan \& Wienen (éds.), Ironie et un peu plus. Hommage à Oswald Ducrot pour son 80ème anniversaire, Francfort, Peter Lang, pp. 65-85.

PROVINE R., SPENCER R., MANDELL D. (2007), «Emotional expression online. Emoticons punctuate website text messages », in Journal of Language and Social Psychology, $\mathrm{n}^{\circ}$ 26, Santa Barbara, University of California, pp. 299-307.

\section{ROSIER L. :}

- (2000), «Interjection, subjectivité, expressivité et discours rapporté à l'écrit : petits effets d'un petit discours », in Cahiers de praxématique, n 34, Montpellier, Université Montpellier III, CNRS, p. 19.

- (mars 2006), « De la vive voix à l'écriture vive : l'interjection et les nouveaux modes d'organisation textuels », in Langages, ${ }^{\circ}$ 161, Paris, Armand Colin, pp. 112-126.

SPERBER D., WILSON D. (1986), La Pertinence, Paris, Minuit.

SWIATKOWSKA M. (2000), Entre dire et faire, De l'interjection, Krakow, Wydunicvwo UJ.

VION R. (2004), «Modalités, modalisations et discours représentés », in Langages, n¹56: Effacement énonciatif et discours rapporté, Paris, Larousse, pp. 96-110.

WITMER D. F., KATZMAN S.L. (1997), « On-line smiles : does gender male a difference in the use of graphic accents ? », in Journal of computer mediated communication, vol. 2, n 4 , Indiana, Indiana University School of Library \& Information Science.

YUS F. (2011), Cyberpragmatics, Internet-mediated communication in context, Amsterdam / Philadelphie, John Benjamins Publishing Company. 